\title{
Serotype Distribution and Resistance Genes Associated with Macrolide and Fluoroquinolone Resistance in Streptococcus agalactiae Isolates from a Hospital in Southern Taiwan
}

\author{
Ying-Hsiang Wang ${ }^{1,2,3}$, Chyi-Liang Chen ${ }^{4}$, Jiun-Nub Hou ${ }^{5}$, Yi-Rou Wang ${ }^{5}$, Ting-Yu Lin ${ }^{5}$, Mei-Hei Wang ${ }^{1}$, \\ Tsung-Han Yang ${ }^{6}$, Chishih $\mathrm{Chu}^{5}$, Cheng-Hsun $\mathrm{Chiu}^{3,4,7}$
}

Background: Antimicrobial resistance of Streptococcus agalactiae (Group B Streptococcus, GBS) has been emerging worldwide. We aimed to examine the correlation of drug-resistant genes with serotypes and with the mutations of the quinolone resistance-determining region (QRDR) in GBS isolates.

Methods: A total of 323 human GBS isolates were collected from a hospital in southern Taiwan. Laboratory investigation included serotyping by a multiplex polymerase chain reaction (PCR) method, antimicrobial susceptibility testing by a disc diffusion method, and mechanism analysis of the resistance to macrolides and fluoroquinolones by PCR and sequencing methods.

Results: $\quad$ Multiplex PCR showed that the most prevalent serotypes were Ib, III, V, and VI, mostly isolated from urine. The ermB gene was highly prevalent in serotypes Ib and V and was associated with clindamycin and macrolide resistance. GBS with a serine-to-leucine mutation at codon 81 in GyrA and with a serine-to-phenylalanine or -tyrosine mutation at codon 79 in ParC had a higher minimum in-

\section{At a Glance Commentary}

Scientific background of the subject

GBS is one of the most common pathogens causing meningitis, bacteremia, and pneumonia with high mortality in neonates, non-pregnant woman, and the elderly. Drug-resistant GBS have been emerging worldwide.

\section{What this study adds to the field}

The most prevalent GBS serotypes in southern Taiwan were Ib, III, V, and VI in this study. The ermB and mefE genes carried in serotypes Ib and V were highly associated with the resistance to clindamycin and macrolides. Mutations within the QRDR region of ParC were determined as genetic factors for high-level fluoroquinolone resistance. hibitory concentration of levofloxacin than isolates with only an aspartic acid-to-tyrosine mutation at codon 83 (>32 $\mu \mathrm{g} / \mathrm{ml} \mathrm{vs.} 16 \mu \mathrm{g} / \mathrm{ml})$ in GyrA.

Conclusions: The most prevalent GBS serotypes were Ib, III, V, and VI. The ermB and mefE genes carried in serotypes $\mathrm{Ib}$ and $\mathrm{V}$ were highly associated with the resistance to macrolides and clindamycin. Mutations at codon 79 and codon 83 of ParC were the major determining factors for high-level fluoroquinolone resistance.

(Biomed J 2015;38:215-220)

Key words: antimicrobial resistance, fluoroquinolone, macrolide, serotype, Streptococcus agalactiae

*C.H. Chiu and C. Chu have equal contributions in this study.

From the ${ }^{1}$ Departments of Pediatrics, Chang Gung Memorial Hospital at Chiayi, Chang Gung University College of Medicine, Taoyuan, Taiwan; ${ }^{2}$ Departments of Laboratory Medicine, Chang Gung Memorial Hospital at Chiayi, Chang Gung University College of Medicine, Taoyuan, Taiwan; ${ }^{3}$ Graduate Institutes of Clinical Medical Sciences, College of Medicine, Chang Gung University, Taoyuan, Taiwan; ${ }^{4}$ Molecular Infectious Diseases Research Center, Chang Gung Memorial Hospital at Linkou, Chang Gung University College of Medicine, Taoyuan, Taiwan; ${ }^{5}$ Department of Microbiology, Immunology, and Biopharmaceuticals, National Chiayi University, Chiayi, Taiwan; ${ }^{6}$ Department of Laboratory Medicine, Chang Gung Memorial Hospital at Linkou, Chang Gung University College of Medicine, Taoyuan, Taiwan; ${ }^{7}$ Division of Pediatric Infectious Diseases, Chang Gung Children's Hospital at Linkou, Chang Gung University College of Medicine, Taoyuan, Taiwan

Received: Feb. 6, 2014; Accepted: Jul. 17, 2014

Correspondence to: Dr. Cheng-Hsun Chiu, Division of Pediatric Infectious Diseases, Chang Gung Children's Hospital at Linkou. 5, Fusing St., Gueishan, Taoyuan 333, Taiwan. Tel: 886-3-3281200 ext. 8896; Fax: 886-3-3288957; E-mail: chchiu@adm.cgmh.org.tw Correspondence to: Prof. Chishih Chu, Department of Microbiology, Immunology, and Biopharmaceuticals, National Chiayi University. 300 University Road, Chiayi 600, Taiwan. Tel: 886-5-2717898; Fax: 886-5-2717831; E-mail: cschu@ mail.ncyu.edu.tw

DOI: $10.4103 / 2319-4170.138306$ 
Ctreptococcus agalactiae (Group B Streptococcus, GBS) $\mathcal{N}$ is one of the most common pathogens causing meningitis, bacteremia, and pneumonia, with high mortality rates in neonates, ${ }^{[1-3]}$ non-pregnant women, the elderly, and immuno-compromised patients. ${ }^{[4,5]}$ To treat GBS infection, penicillin, ampicillin, and cefazolin are the drugs of choice; for patients allergic to penicillin or cephalosporins, vancomycin, macrolides (such as erythromycin, azithromycin, and clarithromycin), and lincosamides (clindamycin) may be used as the alternative drugs. ${ }^{[6-9]}$ Vancomycin resistance has not been reported in GBS. However, in the USA, $12 \%$ of pregnant women are allergic to penicillin, and $25 \%$ and $7 \%$ of GBS isolates from these women are resistant to erythromycin and clindamycin, respectively ${ }^{[8]}$ Resistance to these drugs is higher in isolates from Taiwan; resistance to erythromycin increased from 19 to $46 \%$ and to clindamycin from 18 to $37 \%$ in isolates between 1994 and 1997..10]

The mechanism of macrolide resistance is primarily through acquisition of the resistance genes $\operatorname{ermA}$, ermB, ermC, ermF, ermQ, or ermT, which encode 23S rRNA methylases, ${ }^{[11,12]}$ or mef, which encodes an efflux pump that excludes 14-and 15-membered macrolides from the cell. $^{[6,12,13]}$ In addition, mutations in the quinolone resistance-determining region (QRDR) of GyrA or ParC result in the emergence of fluoroquinolone-resistant GBS. ${ }^{[14,15]}$

Multiplex polymerase chain reaction (PCR) can be used to differentiate GBS isolates into 10 serotypes based on genetic variations in the capsular polysaccharide (CPS) operon and 6 serotypes based on blood agglutination. ${ }^{[13,16-18]}$ Recently, we reported highly erythromycin/clindamycin-resistant phenotypes in different serotypes, with constitutive macrolide-lincosamide-streptogramin $\mathrm{B}\left(\mathrm{CMLS}_{\mathrm{B}}\right)$ as the most prevalent resistance type, followed by inducible macrolide-lincosamide-streptogramin B (iMLS ${ }_{\mathrm{B}}$ ), macrolide (M), and lincosamide-streptogramin A (LSA). ${ }^{[19]}$

In this study, we investigated the prevalence of ermB and $m e f E$ and the association of these genes with different serotypes and antimicrobial resistance. Mutations in the QRDR region of GyrA and ParC in fluoroquinolone-resistant GBS isolates were also determined.

\section{METHODS}

\section{Bacterial sources}

Between 2007 and 2008, 322 S. agalactiae isolates were collected from blood, cerebrospinal fluid (CSF), pus, wounds, urine, vaginal discharge (VA), and other samples from patients treated in Chang Gung Memorial Hospital in Chiayi. After identification, the isolates were maintained on blood agar at $37^{\circ} \mathrm{C}$ and $5 \% \mathrm{CO}_{2}$ for use in future experiments.

\section{Antimicrobial susceptibility test}

The disk diffusion method was used to measure resistance to penicillin (PEN), ceftriaxone (CRO), azithromycin (AZM), erythromycin (ERY), clindamycin (CLI), levofloxacin (LEV), and moxifloxacin (MOX) according to the Clinical and Laboratory Standards Institute (CLSI) standards and guidelines for Streptococcus pneumoniae ATCC49619. ${ }^{[20]}$ The disks $\left(\right.$ Bacto $\left.^{\text {TM }}\right)$ were purchased from Becton, Dickinson and Company (Sparks, MD, USA). The minimum inhibitory concentration (MIC) of LEV and ciprofloxacin (CIP) was determined for each fluoroquinolone-resistant GBS isolate using the Etest ${ }^{\circledR}$ (BioMérieux, Marcy-l'Etoile, France). To ensure accuracy, all isolates tested for PCR and antimicrobial susceptibility were confirmed at least twice for reproducibility.

\section{PCR serotyping and identification of the $\mathrm{ermB}$ and $m e f E$ resistance genes}

GBS isolates were identified by PCR amplification of the $d l t S$ gene and differentiated into 10 serotypes using multiplex PCR, as previously described. ${ }^{[16,17]}$ The primers used to amplify the erm and $m e f E$ genes are listed in Table 1. A protocol for detection of ermB and mefE using multiplex PCR was developed in which 366-and 268-bp PCR products were simultaneously amplified and then separated on a $1.5 \%$ agarose gel. After staining with ethidium bromide (EtBr), images were recorded under UV illumination.

\section{Sequencing of the QRDR region of gyrA and parC}

The QRDR region of the gyrA and parC genes from five fluoroquinolone-resistant isolates and two fluoroquino-

Table 1: Primers used to amplify ermB, mefE, and the QRDR regions of the $\operatorname{gyr} A$ and $\operatorname{par} C$ genes

\begin{tabular}{cllcc}
\hline Gene & Primer & Sequence $\left(5^{\prime} \rightarrow 3^{\prime}\right)$ & $\begin{array}{c}\mathrm{T} \\
\left({ }^{\circ} \mathrm{C}\right)\end{array}$ & $\begin{array}{c}\text { Product } \\
\text { size }(\mathrm{bp})\end{array}$ \\
\hline ermB & ermB-F & GCATTTAACGACGAAACTGGCT & 54 & 366 \\
& ermB-R & GAAAGCATTCCGCTGGCAGCT & & \\
ermC & ermC-F & TGAAATCGGCTCAGGAAAAGG & 54.4 & 80 \\
& ermC-R & GTCTATTCAATGGCAGTTACG & & \\
ermF & ermF-F & GCATACCTTTGTTCCTCGGT & 54.4 & 196 \\
& ermF-R & GAGGTGAATACTTCTTGAGTGC & & \\
ermQ & ermQT-F & ATTGGTCCAGGAAAAGGTCAT & & \\
& ermQ-R & CTAGCCACATATCAGTTGGT & 53 & 274 \\
ermT & ermT-R & GATGCAGTTTATGCACCCCT & 54.4 & 651 \\
mefE & mefE-F & AAACAGGATCTGCGATGGTCT & 54 & 268 \\
& mefE-R & CGGAGTATAAGAGTGCTGCA & & \\
parC & parC-F & CCTTGAATGATAGCGCCAGT & 45 & 575 \\
& parC-R & GTTGCCGGATATTCGTGATG & & \\
gyrA & gyrA-F & CGCCATGAGTGTCATTGTTG' & 52 & 452 \\
& gyrA-R & CAATACCAGTTGCACCATTGAC & & \\
\hline Abbreviation: QRDR Quinolone resistance-determining region &
\end{tabular}

Abbreviation: QRDR: Quinolone resistance-determining region 
lone-susceptible isolates was amplified using the primers listed in Table 1. The PCR products were purified using the Wizard PCR Prep DNA Purification System (Promega, Madison, WI, USA). The purified PCR products were sequenced using an ABI3730 autosequencer. The sequences were analyzed using the SeqMan and Megalign programs in the Lasergene software (DNASTAR, Inc., Madison, WI, USA).

\section{RESULTS}

\section{Clinical isolates and serotype analysis}

Majority (91.9\%) of the 322 GBS isolates were associated with non-invasive infection, with urine (73.9\%) being the most frequent. A substantial amount (8.1\%) of invasive infections was found, including 25 bacteremia cases. Overall, eight serotypes (Ia, Ib, and II-VII) were identified. The prevalence ranged from $1.9 \%$ for serotype Ia to $35.7 \%$ for serotype $V$ [Table 2]. Serotype distribution did not differ significantly between invasive and non-invasive infections. However, serotypes Ia and VII were not found in our invasive isolates.

\section{Antimicrobial susceptibility}

All isolates were susceptible to the $\beta$-lactams PEN and $\mathrm{CRO}$, and only five isolates were resistant to fluoroquinolone. The prevalence of resistance to AZM, ERY, and CLI was $56.2 \%, 53.1 \%$, and $52.5 \%$, respectively [Table 3].

\section{Prevalence of the ermB and mefE genes}

Although five erm genes (ermB, ermC, ermF, erm Q, and erm $T$ ) were examined in this study, only ermB was identified in detail. We developed a multiplex PCR protocol using primers for both $e r m B$ and mefE that could amplify simultaneously 366-bp and 268-bp PCR products from ermB and $m e f E$, respectively. The prevalence of the GBS isolates carrying ermB and mefE was $68.0 \%$ and $1.9 \%$, respectively, while $2.8 \%$ of the isolates possessed both ermB and $m e f E$; however, $27.3 \%$ of the isolates lacked both ermB and $m e f E$ (ermB $B^{-}$mefE $E^{-}$[ [Table 3].

Regarding the association of resistance genes with antimicrobial resistance, we found that $75.8 \%$ of the erm $B^{+}$isolates were resistant to each of the three drugs, indicating that the erm $B$ gene may be the major factor associated with the resistance to the three drugs in GBS isolates. However, the ermB and mefE genes were not the only determinants responsible for the resistance to AZM, ERY, and CLI in GBS isolates. We observed that $24.2 \%$ of the $e r m B^{+}$isolates and $33.3 \%$ of the $\mathrm{ermB}^{+} \mathrm{mefE}^{+}$isolates were susceptible to all three drugs. In contrast, $85.2 \%$ of the erm $B^{-} m e f E^{-}$isolates were susceptible to all three drugs, while $14.8 \%$ of the $e r m B^{-} m e f E^{-}$isolates were resistant to at least one of the three drugs [Table 3].
Table 2: Prevalence of each serotype of GBS isolates associated with sources of isolation

\begin{tabular}{lcccccc}
\hline Serotype & \multicolumn{2}{c}{$\begin{array}{c}\text { Total, } \\
n(\%)\end{array}$} & \multicolumn{4}{c}{ Source, $n(\%)$} \\
\cline { 4 - 7 } & \multicolumn{2}{c}{$n$} & \multicolumn{2}{c}{ Invasive } & \multicolumn{2}{c}{ Non-invasive } \\
\hline Ia & 6 & $(1.9)$ & 0 & $(0.0)$ & 6 & $(100.0)$ \\
Ib & 65 & $(20.2)$ & 6 & $(9.2)$ & 59 & $(90.8)$ \\
II & 11 & $(3.4)$ & 1 & $(9.1)$ & 10 & $(90.9)$ \\
III & 43 & $(13.4)$ & 3 & $(7.0)$ & 40 & $(93.0)$ \\
IV & 32 & $(9.9)$ & 2 & $(6.3)$ & 30 & $(93.8)$ \\
V & 115 & $(35.7)$ & 10 & $(8.7)$ & 105 & $(91.3)$ \\
VI & 45 & $(14.0)$ & 4 & $(8.9)$ & 41 & $(91.1)$ \\
VII & 5 & $(1.6)$ & 0 & $(0.0)$ & 5 & $(100.0)$ \\
Total & 322 & $(100.0)$ & 26 & $(8.1)$ & 296 & $(91.9)$ \\
\hline Abbryyyyy & & & & & &
\end{tabular}

Abbreviations: Invasive: Blood, cerebrospinal fluid, urine; Non-invasive: Pus, wound, vaginal discharge; GBS: Group B Streptococcus

Table 3: Association of the azithromycin, erythromycin, and clindamycin resistance-associated genes $\operatorname{ermB}$ and $m e f E$ with resistance phenotypes in GBS isolates

\begin{tabular}{lccccc}
\hline $\begin{array}{l}\text { Resistance } \\
\text { gene }\end{array}$ & Azithromycin Erythromycin Clindamycin & \multicolumn{2}{c}{$\begin{array}{c}\text { Total } \\
(\%)\end{array}$} \\
\hline ermB+ & $\mathrm{S}$ & $\mathrm{S}$ & $\mathrm{R}$ & 4 & $219(68.0)$ \\
& $\mathrm{S}$ & $\mathrm{R}$ & $\mathrm{S}$ & 3 & \\
& $\mathrm{R}$ & $\mathrm{S}$ & $\mathrm{R}$ & 4 & \\
& $\mathrm{R}$ & $\mathrm{R}$ & $\mathrm{S}$ & 7 & \\
& $\mathrm{R}$ & $\mathrm{R}$ & $\mathrm{R}$ & 148 & \\
mefE+ & $\mathrm{S}$ & $\mathrm{S}$ & $\mathrm{S}$ & 53 & \\
& $\mathrm{R}$ & $\mathrm{S}$ & $\mathrm{S}$ & 1 & $6(1.9)$ \\
& $\mathrm{R}$ & $\mathrm{R}$ & $\mathrm{S}$ & 4 & \\
ermB+mefE++ & $\mathrm{S}$ & $\mathrm{R}$ & $\mathrm{R}$ & 1 & \\
& $\mathrm{~S}$ & $\mathrm{R}$ & $\mathrm{S}$ & 1 & $9(2.8)$ \\
& $\mathrm{R}$ & $\mathrm{R}$ & $\mathrm{S}$ & 2 & \\
& $\mathrm{R}$ & $\mathrm{R}$ & $\mathrm{R}$ & 3 & \\
ermB mefE & $\mathrm{S}$ & $\mathrm{S}$ & $\mathrm{S}$ & 3 & \\
& $\mathrm{R}$ & $\mathrm{S}$ & $\mathrm{S}$ & 2 & $88(27.3)$ \\
& $\mathrm{S}$ & $\mathrm{S}$ & $\mathrm{R}$ & 1 & \\
& $\mathrm{R}$ & $\mathrm{S}$ & $\mathrm{R}$ & 2 & \\
& $\mathrm{R}$ & $\mathrm{R}$ & $\mathrm{S}$ & 2 & \\
& $\mathrm{R}$ & $\mathrm{R}$ & $\mathrm{R}$ & 6 & \\
Total $n(\%)$ & $\mathrm{S}$ & $\mathrm{S}$ & $\mathrm{S}$ & 75 & \\
& 181 & $(56.2)$ & $1715(3.1)$ & $169(52.5)$ & $322(100)$
\end{tabular}

Abbreviations: S: Susceptible; R: Resistant; GBS: Group B Streptococcus

\section{Association of resistance genes with serotypes}

The ratio of the $e r m B^{+}$number to the $e r m B^{-}$mefE $E^{-}$number was then used to reflect the susceptibility to the three drugs in GBS isolates. The ratio was 2.49 (219/88) in total. However, the ratio differed among serotypes; a ratio of $2(4 / 2)$ was shown in serotype Ia, 20.3 (62/3) in serotype Ib, 1.74 (7/4) in serotype II, 1.5 (22/15) in serotype III, $0.87(13 / 15)$ in serotype IV, $3.1(84 / 27)$ in serotype V, $1.5(26 / 18)$ in serotype VI, and a ratio of $0.25(1 / 4)$ in serotype VII [Table 4]. The results showed that all serotypes (with the erm $B^{+} / e r m B^{-} m e f E^{-}$ratio of $>1$ ), except 
serotypes IV and VII, had higher rates of resistance to the three drugs.

\section{Fluoroquinolone resistance-associated mutations in the QRDR regions of GyrA and ParC}

Sequence analysis revealed that all LEV- and MOX-resistant isolates carried an identical serine-to-leucine mutation (S81L) at codon 81 of GyrA and one of two separate mutations in ParC: A serine-to-tyrosine mutation (S79Y) at codon 79 or an aspartic acid-to-phenylalanine (D83F) or aspartic acid-to-tyrosine mutation (D83Y) at codon 83 [Table 5]. The MICs of LEV were $16 \mu \mathrm{g} /$ $\mathrm{ml}$ for the isolates with a mutation at codon 79 of ParC and $>32 \mu \mathrm{g} / \mathrm{ml}$ for the isolates with a mutation at codon 83 of ParC. The mutations in LEV- and MOX-resistant isolates did not affect the MIC of CIP at the tested concentrations (greater than $32 \mu \mathrm{g} / \mathrm{ml}$ ), but they did affect the MIC of LEV.

Table 4: Prevalence of $e r m B$ and $m e f E$ in different serotypes of GBS isolates

\begin{tabular}{|c|c|c|c|c|c|c|}
\hline \multirow[t]{2}{*}{ Serotype } & \multirow[t]{2}{*}{ Number } & \multirow{2}{*}{$\begin{array}{c}\text { Prevalence, } \\
\%\end{array}$} & \multicolumn{4}{|c|}{ Resistance gene, $n(\%)$} \\
\hline & & & $e r m B^{+}$ & $m e f E^{+}$ & $\begin{array}{l}\text { ermB } B^{+} \\
\text {mefE }\end{array}$ & $\begin{array}{l}\text { ermB } \\
\text { mefE }\end{array}$ \\
\hline Ia & 6 & 1.9 & $4(66.7)$ & $0(0)$ & $0(0)$ & $2(33.3)$ \\
\hline $\mathrm{Ib}$ & 65 & 20.2 & $62(95.3)$ & $0(0)$ & $0(0)$ & $3(4.7)$ \\
\hline II & 11 & 3.4 & 7 (63.6) & $0(0)$ & $0(0)$ & $4(36.4)$ \\
\hline III & 43 & 13.4 & $22(51.2)$ & $3(7.0)$ & $3(7.0)$ & $15(34.9)$ \\
\hline IV & 9.9 & 32 & $13(40.6)$ & $9.4(3)$ & $1(3.1)$ & $15(46.9)$ \\
\hline V & 115 & 35.7 & $84(73.0)$ & $0(0)$ & $4(3.5)$ & $27(23.5)$ \\
\hline VI & 45 & 14.0 & $26(57.8)$ & $0(0)$ & $1(2.2)$ & $18(40.0)$ \\
\hline VII & 5 & 1.6 & $1(20.0)$ & $0(0)$ & $0(0)$ & $4(80.0)$ \\
\hline Total & 322 & 100 & 219 (68.0) & $6(1.9)$ & $9(2.8)$ & 88 (27.3) \\
\hline
\end{tabular}

Abbreviation: GBS: Group B Streptococcus

Table 5: Minimum inhibitory concentrations of levofloxacin and ciprofloxacin and mutations in the QRDR of GyrA and $\operatorname{ParC}$ in fluoroquinolone-resistant GBS isolates

\begin{tabular}{|c|c|c|c|c|c|c|c|}
\hline \multirow[t]{2}{*}{ Isolates } & \multicolumn{2}{|c|}{ Resistance to } & \multicolumn{2}{|c|}{$\mathrm{MIC}(\mu \mathrm{g} / \mathrm{ml})$} & \multicolumn{3}{|c|}{ Mutation in QRDR region of } \\
\hline & LEV & MOX & LEV & CIP & GyrA & ParC & \\
\hline G25* & $\mathrm{S}$ & $\mathrm{S}$ & 0.5 & 0.5 & $\mathrm{~S}_{81}$ & $\mathrm{~S}_{79}$ & $\mathrm{D}_{83}$ \\
\hline G39* & $\mathrm{S}$ & $S$ & 0.5 & 0.5 & $\mathrm{~S}_{81}$ & $\mathrm{~S}_{79}$ & $\mathrm{D}_{83}$ \\
\hline G15 & $\mathrm{R}$ & $\mathrm{R}$ & 16 & $>32$ & S81L & $\mathrm{S}_{79}$ & D83Y \\
\hline G22 & $\mathrm{R}$ & $\mathrm{R}$ & 16 & $>32$ & S81L & $\mathrm{S}_{79}$ & D83Y \\
\hline G57 & $\mathrm{R}$ & $\mathrm{R}$ & $>32$ & $>32$ & S81L & S79F & $\mathrm{D}_{83}$ \\
\hline G233 & $\mathrm{R}$ & $\mathrm{R}$ & 16 & $>32$ & S81L & $\mathrm{S}_{79}$ & D83Y \\
\hline G309 & $\mathrm{R}$ & $\mathrm{R}$ & $>32$ & $>32$ & S81L & S79Y & $\mathrm{D}_{83}$ \\
\hline
\end{tabular}

*The G25 and G39 strains are fluoroquinolone-sensitive GBS isolates and used as a control. Abbreviations: CIP: Ciprofloxacin; LEV: Levofloxacin; MOX: Moxifloxacin; S: Susceptible; R: Resistant; D: Aspartic acid; F: Phenylalanine; L: Leucine; S: Serine; Y: Tyrosine, GBS: Group B Streptococcus, QRDR: Quinolone resistance-determining region

\section{DISCUSSION}

S. agalactiae is a zoonotic pathogen of fish, bovines, and humans. ${ }^{[19,21,22]}$ Most GBS isolates were collected from female patients. ${ }^{[19]}$ The predominant human serotype differs between countries, with serotype $\mathrm{V}$ being the primary serotype in the USA, ${ }^{[21,23]}$ serotype III in France and Zimbabwe, and serotypes III and V being the primary serotypes in Korea. ${ }^{[16,24,25]}$ In this study, although the total number of GBS isolates decreased from 2007 to 2008 , the predominant serotypes varied. Serotype V, serotype Ib, and serotype III were predominant in 2007; however, serotype V, serotype VI, and serotype Ib were prevalent in 2008. Although the present study was performed in only one hospital, the data appeared to suggest that serotype $\mathrm{V}$ is gradually becoming the dominant serotype, as it is in the USA and Korea.

In a previous study, we determined that the predominant ERY/CLI resistance phenotype was iMLS $_{\mathrm{B}}$ in serotype $\mathrm{Ib}$ and $\mathrm{CMLS}_{\mathrm{B}}$ in serotypes III and $\mathrm{V}^{[19]}$ The prevalent resistance genes vary between countries, with ermB, ermTR, and mefA being prevalent in Spain and the USA ${ }^{[26]}$ and ermB and $\ln u B$ being prevalent in Korea. ${ }^{[25]}$ The association of the erm and mef drug resistance genes with drug resistance phenotype and serotype was seldom reported. In Korea, ermB was frequently identified in serotypes III and IV. ${ }^{[27]}$ Nevertheless, in this study, ermB was primarily identified in serotypes Ib and V, and mefE in serotypes III and IV [Table 4], implicating that these two drug resistance genes are frequently transferred between serotypes through lateral gene transfer. ${ }^{[28,29]}$ Further investigation is required to determine whether serotype switching is involved in the observed differences in ermB-associated serotypes. ${ }^{[30]}$

The prevalence of CLI and ERY resistance in the tested isolates differed ${ }^{[25,27]}$ and $e r m B$ was present in $91.9 \%$ of ERY-resistant isolates and in $84.0 \%$ of CLI-resistant isolates in Korea ${ }^{[25]}$ However, in this study, ermB was present in those isolates with a higher population resistant to AZM (90.6\%), ERY (95.9\%), or CLI (94.1\%). In addition, $14.8 \% \mathrm{ermB}^{-} \mathrm{mefE}^{-}$isolates were still resistant to one of these drugs. These results indicate that not only ermB is the major gene responsible for resistance to these three drugs,

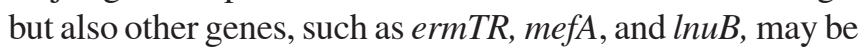
involved, as is the case in Korea. ${ }^{[25,26]}$ We also determined that 57 isolates that possessed $\mathrm{erm} B$ or $m e f E$ were susceptible to all three drugs, indicating the possibility that mutations in these genes inhibit the function of the encoded proteins.

Since the discovery of fluoroquinolone-resistant GBS isolates carrying a serine-to-leucine mutation at codon 81 of GyrA and a serine-to-phenylalanine mutation at codon 79 of ParC in 2003, ${ }^{[14,15,31]}$ an aspartic acid-to-tyrosine mutation at codon 83 of ParC was also identified in fluoroquinolone-resistant GBS isolates. ${ }^{[32]}$ Isolates with double mutations at 
codon 81 of GyrA and codon 79 and/or codon 83 of ParC had been reported to have the same MIC $(>32 \mu \mathrm{g} / \mathrm{ml})$ of LEV. ${ }^{[14,15,31,32]}$ However, in this study, differences in the MICs of the tested drugs for fluoroquinolone-resistant GBS isolates with mutations in ParC were observed. With the identical mutation at codon 81 of GyrA, the fluoroquinolone-resistant isolates with the second mutation of a serine-to-phenylalanine or -tyrosine at codon 79 of ParC had an at least twofold higher MIC than those with the second mutation of a serine-to-tyrosine at codon 83 of ParC. Because these mutations are similar, with a change to tyrosine at codon 79 or codon 83, the variations in MIC for LEV may be due to positional conformation change.

\section{Conclusions}

The most prevalent GBS serotypes Ib, III, V, and VI were primarily isolated from urine samples. CLI and macrolide (AZM and ERY) resistance were highly associated with the presence of the $\operatorname{erm} B$ gene. The resistance genes ermB and $m e f E$ were associated with specific serotypes $\mathrm{Ib}$ and V. At least a twofold increase was observed in the MIC of LEV between isolates carrying mutations at codon 79 and codon 83 of ParC.

\section{Acknowledgment}

This work was supported by grantNSC98-2321-B-415-003 from the National Science Council of Executive Yuan, Taiwan.

\section{REFERENCES}

1. Berg S, Trollfors B, Lagergard T, Zackrisson G, Claesson BA. Serotypes and clinical manifestations of group B streptococcal infections in western Sweden. Clin Microbiol Infect 2000;6:9-13.

2. Majm TS, Adriaanse AH, Gerards LJ, Kimoen LL. Strategy to prevent neonatal early-onset group B streptococcal disease in the Netherlands. Rev Med Microbiol 2003;14:35-9.

3. Poyart C, Réglier-Poupet H, Tazi A, Billoët A, Dmytruk N, Bidet P, et al. Invasive group B streptococcal infections in infants, France. Emerg Infect Dis 2008;14:1647-9.

4. Huang PY, Lee MH, Yang CC, Leu HS. Group B streptococcal bacteremia in non-pregnant adults. J Microbiol Immun Infect 2005;39:237-41.

5. Savoia D, Gottimer C, Crocilla C, Zucca M. Streptococcus agalactiae in pregnant women: Phenotypic and genotypic characters. J Infect 2008;56:120-5.

6. de Azavedo JC, McGavin M, Duncan C, Low DE, Mcgeep A. Prevalence and mechanisms of macrolide resistance in invasive and noninvasive group B Streptococcus isolates from Ontario, Canada. Antimicrob Agents Chemother 2001;45:3504-8.

7. von Both U, Buerckstuemmer A, Fluegge K, Berner R. Heterogeneity of genotype-phenotype correlation among macrolide-resistant Streptococcus agalactiae isolates. Antimicrob Agents Chemother 2005;49:3080-2.
8. Chohan L, Hollier LM, Bishop K, Kilpatrick CC. Patterns of antibiotic resistance among group B Streptococcus isolates: 2001-2004. Infect Dis Obstet Gynecol 2006;2006:57492.

9. Heelan JS, Hasenbein ME, McAdam AJ. Resistance of group B Streptococcus to selected antibiotics, including erythromycin and clindamycin. J Clin Microbiol 2004;42:1263-4.

10. Hsueh PR, Teng LJ, Lee LN, Ho SW, Yang PC, Luh KT. High incidence of erythromycin resistance among clinical isolates of Streptococcus agalactiae in Taiwan. Antimicrob Agents Chemother 2001;45:3205-8.

11. Roberts MC. Location of rRNA methylases in the published literature. In: Bonomo RA, Tolmasky M, editors. Enzyme-Mediated Resistance to Antibiotic: Mechanisms, Dissemination, and Prospects for Inhibition. Washington, D.C.: ASM Press; 2007. p. 53-66.

12. DiPersio LP, DiPersio JR, Frey KC, Beach JA. Prevalence of the erm $(T)$ gene in clinical isolates of erythromycin-resistant group D Streptococcus and Enterococcus. Antimicrob Agents Chemother 2008;52:1567-9.

13. Gygax SE, Schuyler JA, Kimmel LE, Trama JP, Mordechai E, Adelson ME. Erythromycin and clindamycin resistance in group B streptococcal clinical isolates. Antimicrob Agents Chemother 2006;50:1875-7.

14. Wehbeh W, Roberto RD, Li X, Mariano N, Grenner L, Sorana SM, et al. Fluoroquinolone- resistant Streptococcus agalactiae: Epidemiology and mechanism of resistance. Antimicrob Agents Chemother 2005;49:2495-7.

15. Wu HM, Janapatla RP, Ho YR, Hung KH, Wu CW, Yan JJ, et al. Emergence of fluoroquinolone resistance in group B streptococcal isolates in Taiwan. Antimicrob Agents Chemother 2008;52:1888-90.

16. Poyart C, Tazi A, Reglier-Poupet H, Billoet A, Tavares N, Raymond J, et al. Multiplex PCR assay for rapid and accurate capsular typing of group B streptococci. J Clin Microbiol 2007;45:1985-8.

17. Slotved HC, Kong F, Lambertsen L, Sauer S, Gilbert GL. Serotype IX, a proposed new Streptococcus agalactiae serotype. J Clin Microbiol 2007;45:2929-36.

18. Cieslewicz MJ, Chaffin D, Glusman G, Kasper D, Madan A, Rodrigues S, et al. Structural and genetic diversity of group B Streptococcus capsular polysaccharides. Infect Immun 2005;73:3096-103.

19. Wang YH, Su LH, Hou JN, Yang TH, Lin TY, Chu C, et al. Group B streptococcal disease in nonpregnant patients: Emergence of highly resistant strains of serotype Ib in Taiwan in 2006 to 2008. J Clin Microbiol 2010;48:2571-4.

20. CLSI. Performance standards for antimicrobial susceptibility tests; approved standard, $12^{\text {th }}$ ed. M100-S20-U. Clinical and Laboratory Standards Institute. Wayne, Pennsylvania, USA, 2010.

21. Dogan B, Schukken YH, Santisteban C, Boor KJ. Distribution of serotypes and antimicrobial resistance genes among Streptococcus agalactiae isolates from bovine and human hosts. J Clin Microbiol 2005;43:5899-906.

22. Foxman B, Gillespie BW, Manning SD, Marrs CF. Risk factors for group B streptococcal colonization: Potential for different transmission systems by capsular type. Ann Epidemiol 2007; 17:854-62.

23. Ulett KB, Benjamin WH Jr, Zhuo F, Xiao M, Kong F, Gilbert GL, et al. Diversity of group B Streptococcus serotypes causing urinary tract infection in adults. J Clin Microbiol 2009;47:2055-60. 
24. Mavenyengwa RT, Maeland JA, Moyo SR. Distinctive features of surface-anchored proteins of Streptococcus agalactiae strains from Zimbabwe revealed by PCR and dot blotting. Clin Vaccine Immunol 2008;15:1420-4.

25. Seo YS, Srinivasan U, Oh KY, Shin JH, Chae JD, Kim MY, et al. Changing molecular epidemiology of group B Streptococcus in Korea. J Korean Med Sci 2010;25:817-23.

26. Gonzalez JJ, Andreu A, the Spanish Group for the Study of Perinatal Infection from the Spanish Society for Clinical Microbiology and Infectious Diseases. Multicenter study of the mechanisms of resistance and clonal relationships of Streptococcus agalactiae isolates resistant to macrolides, lincosamides, and ketolides in Spain. Antimicrob Agents Chemother 2005;49:2525-7.

27. Uh Y, Kim HY, Jang IH, Hwang GY, Yoon KJ. Correlation of serotypes and genotypes of macrolide-resistant Streptococcus agalactiae. Yonsei Med J 2005;46:480-3.

28. Del Grosso M, Northwood JG, Farrell DJ, Pantosti A. The macrolide resistance genes erm $(B)$ and mef $(E)$ are carried by Tn2010 in dual-gene Streptococcus pneumoniae isolates belonging to clonal complex CC271. Antimicrob Agents Chemother 2007;51:4184-6.

29. Shiojima T, Fujiki Y, Sagai H, Iyobe S, Morikawa A. Prevalence of Streptococcus pneumoniae isolates bearing macrolide resistance genes in association with integrase genes of conjugative transposons in Japan. Clin Microbiol Infect 2005;11:808-13.

30. Martins ER, Melo-Cristino J, Ramirez M. Evidence for rare capsular switching in Streptococcus agalactiae. J Bacteriol 2010;192:1361-9.

31. Kawamura Y, Fujiwara H, Mishima N, Tanaka Y, Tanimoto A, Ikawa S, et al. First Streptococcus agalactiae isolates highly resistant to quinolones, with point mutations in gyrA and parC. Antimicrob Agents Chemother 2003;47:3605-9.

32. Biedenbach DJ, Toleman MA, Walsh TR, Jones RN. Characterization of fluoroquinolone-resistant beta-hemolytic Streptococcus spp. isolated in North America and Europe including the first report of fluoroquinolone-resistant Streptococcus dysgalactiae subspecies equisimilis: Report from the SENTRY Antimicrobial Surveillance Program (1997-2004). Diagn Microbiol Infect Dis 2006;55:119-27. 